\title{
SELETIVIDADE ALIMENTAR E INFLUÊNCIA DA IDADE DA FOLHA DE EUCALYPTUS SPP. PARA THYRINTEINA ARNOBIA (LEPIDOPTERA: GEOMETRIDAE) ${ }^{1}$
}

\author{
RAIMUNDANONATA SANTOS LEMOS², WILSON BADIALI CROCOMO, LUIZ CARLOS FORTI \\ e CARLOS FREDERICO WILCKEN ${ }^{3}$
}

\begin{abstract}
RESUMO - Thyrinteina arnobia (Stoll, 1782) (Lepidoptera: Geometridae) é considerada uma das mais sérias pragas do eucalipto no Brasil. Este trabalho foi realizado com o objetivo de estudar a preferência alimentar de T. arnobia em seis espécies de eucalipto e a influência da idade foliar sobre a seleção hospedeira, utilizando-se folhas jovens e velhas de Eucalyptus grandis, Eucalyptus camaldulensis, Eucalyptus saligna, Eucalyptus citriodora, Eucalyptus robusta e Eucalyptus cloeziana. Lagartas de T. arnobia alimentadas na geração anterior com folhas de E. grandis preferiram folhas jovens de $E$. grandis e E.cloeziana, enquanto as alimentadas com E. saligna, na geração anterior, preferiram folhas velhas de E. grandis. A espécie preferida por lagartas de T. arnobia foi E. grandis, observando-se, também maior preferência por folhas jovens que por folhas velhas nas espécies utilizadas nos testes.
\end{abstract}

Termos para indexação: lagarta-desfolhadora, eucalipto, seleção hospedeira.

\section{FEEDING SELECTIVITY AND INFLUENCE OF LEAF AGE OF EUCALYPTUS SPP FOR THYRINTEINA ARNOBIA (LEPIDOPTERA: GEOMETRIDAE)}

\begin{abstract}
Thyrinteina arnobia (Stoll, 1782) (Lepidoptera: Geometridae) is considered one of the most serious Eucalyptus pests in Brazil. This work was carried out aiming to study feeding preference of T. arnobia and the influence of leaf age on the host selection, using young and old leaves of Eucalyptus grandis, Eucalyptus camaldulensis, Eucalyptus saligna, Eucalyptus citriodora, Eucalyptus robusta e Eucalyptus cloeziana. Caterpillars fed with E. grandis leaves in previous generation, preferred young leaves of E. grandis and E. cloeziana, while caterpillars fed with E. saligna in previous generation preferred old leaves of E. grandis. The most consumed species was E. grandis, and the caterpillars preferred young leaves than old ones, for all species studied
\end{abstract}

Index terms: defoliator caterpillar, eucalyptus, host selection.

\section{INTRODUÇÃo}

Thyrinteina arnobia (Stoll, 1782) (Lepidoptera: Geometridae) tem sido considerada o lepidóptero desfolhador mais danoso para a eucaliptocultura bra-

\footnotetext{
${ }^{1}$ Aceito para publicação em 25 de junho de 1998.

${ }^{2}$ Enga Agra - Dra - , Auxiliar de Ensino, Dep. de Fitotecnia e Fitossanidade, CCA/UEMA, Caixa Postal 9, CEP 65055-580 São Luis, MA. E-mail: rlemos@cca.uema.br

${ }^{3}$ Eng. Agr., Dr., Prof. Assistente, Dep. de Defesa Fitossanitária, FCA/UNESP, Caixa Postal 237, CEP18603-970 Botucatu, SP. E-mail: wcrocomo@fca.unesp.br
}

sileira. Embora alguns trabalhos tenham confirmado a ocorrência de vários graus de resistência de espécies e procedências de eucalipto a formigas cortadeiras, pouco se sabe a esse respeito, em relação a lepidópteros desfolhadores.

Osse \& Briquelot (1970) verificaram que Eucalyptus paniculata mostrou-se mais suscetível ao ataque de T. arnobia do que Eucalyptus tereticornis, Eucalyptus alba e Eucalyptus robusta. Na região de João Pinheiro, $\mathrm{MG}$, esse inseto desfolhou severamente Eucalyptus grandis, Eucalyptus saligna, Eucalyptus urophylla, Eucalyptus cloeziana e Eucalyptus citriodora, sendo que as árvores de Eucalyptus camaldulensis presentes na área não foram afetadas (Anjos et al., 1987). 
Oliveira et al. (1984), estudando o comportamento de algumas espécies de eucalipto em laboratório, verificaram que Tryrinteina arnobia apresentou alta preferência por E. saligna e E. grandis, e baixa preferência por E. camaldulensis.

A maioria das espécies de eucalipto contêm compostos secundários, particularmente óleos essenciais (Penfold \& Willis, 1961) e fenóis, incluindo os taninos (Hills, 1966; Fox \& Macauley, 1977). Em florestas temperadas o desfolhamento por herbívoros é abundante em folhas jovens. As folhas velhas podem conter substâncias deletérias, como taninos, ou ser mais duras, porém não está bem claro, se esses efeitos são devidos às substâncias secundárias das plantas ou meramente ao valor nutritivo mais baixo das folhas velhas.

Drooz (1970), trabalhando com Ennomos subsignarius (Lepidoptera: Geometridae), verificou que este inseto também tem sua biologia afetada em decorrência da idade foliar de Quercus rubra (L.) e de Caria glabra (Miller). Peres Filho (1989) verificou que lagartas recém-eclodidas de T. arnobia preferiram alimentar-se em folhas jovens de $E$. grandis e que o desenvolvimento da fase larval e o peso das pupas são afetados pela idade das folhas.

Este trabalho objetivou estudar a preferência alimentar de Tryrinteina arnobia em seis espécies de eucalipto e a influência da idade foliar sobre a seleção hospedeira.

\section{MATERIAL E MÉTODOS}

Este trabalho foi realizado no Laboratório de Entomologia, da Faculdade de Ciências Agronômicas/ UNESP, Botucatu, SP, a $25 \pm 2{ }^{\circ} \mathrm{C}, 70-80 \%$ UR e fotoperíodo de 12 horas.

Foram realizados dois experimentos. No primeiro, foram utilizados 100 tubos de plástico contendo quatro lagartas de T. arnobia cada um, que foram alimentadas com folhas de E. grandis na geração anterior. Da mesma forma o segundo experimento foi conduzido, em que as lagartas foram alimentadas com E. saligna. As lagartas descendentes desses experimentos foram submetidas aos testes de preferência alimentar, utilizando-se como tratamentos folhas jovens (retiradas da extremidade apical dos ramos da parte superior da copa) e folhas velhas (retiradas da base dos ramos da parte inferior da copa) de seis espécies de eucalipto: E. grandis (procedência Anhembi), E. saligna (Itatinga), E.camaldulensis (Anhembi), E. citriodora (Ouriçangas), E. cloeziana (Acesita) e E. robusta (Anhembi), coletadas em plantios com idade de um ano, situados no Campus da FCA/ UNESP/Botucatu, SP.

Os testes de preferência foram realizados separadamente, um com folhas jovens e outro com folhas velhas, das seis espécies de eucalipto já citadas. Como arenas, foram usadas bandejas de plástico de $25 \mathrm{~cm}$ de diâmetro por $3 \mathrm{~cm}$ de altura, com o fundo revestido por uma fina camada de gesso, que era umedecida antes de cada teste, a fim de facilitar a fixação das folhas de eucalipto e conservá-las durante o período de avaliação. No centro das arenas foram liberadas 30 lagartas, e cada arena continha seis folhas inteiras de eucalipto, ou seja, uma de cada espécie distribuída ao acaso. Foram utilizadas seis bandejas por ensaio, sendo cada uma considerada como uma repetição.

Ante a dificuldade de medir a área foliar consumida por T. arnobia nos primeiros ínstares, optou-se por realizar os testes de preferência alimentar do $3 \stackrel{0}{0}, 4^{0}$ e $5 \underline{0}$ ínstar, ficando também de fora as lagartas do 60 ínstar, uma vez que nesta fase, além do consumo de área foliar ser maior, poderia ocorrer a pupação durante a realização dos testes. Após a colocação das lagartas, as bandejas foram vedadas com película de PVC (Magipack).

Antes de serem oferecidas às lagartas, as folhas foram lavadas em água corrente e secadas com papel toalha. Para determinação da área consumida, em centímetros quadrados, as folhas das seis espécies de eucalipto foram previamente desenhadas em papel sulfite, e a cada avaliação a área foliar consumida era demarcada no seu respectivo contorno, com cores diferentes em cada período de avaliação, que foi de 2, 4, 6, 10 e 24 horas. Dessa forma, calculou-se a área foliar ingerida pelas lagartas, baseando-se na relação peso/área do papel. As pesagens foram realizadas em balança analítica com precisão de 0,0001 g. Nos testes de preferência alimentar, foi realizada a prova não-paramétrica de Friedman, com cálculo da estatística $\chi^{2}$, para a comparação de espécies de eucalipto, e a prova de Wilcoxon, para comparar o consumo de folhas jovens e velhas em cada espécie (Zar, 1984).

\section{RESULTADOS E DISCUSSÃO}

Diversos autores têm observado que, geralmente, as folhas jovens são mais nutritivas que as folhas velhas pelo fato de conterem baixos níveis de substâncias repelentes ou tóxicas (Raupp \& Denno, 1983; Meyer \& Montgomery, 1987). O consumo de folhas jovens por lagartas de T. arnobia foi maior no 
3o e 4 o ínstar (Tabelas 1 e 2), o que sugere que as lagartas, nestes ínstares, preferem folhas jovens, quando alimentadas com E. grandis na geração anterior. Drooz (1970) admitiu que lagartas de Ennomos subsignarius no $1^{\circ}$ e $4^{\circ}$ ínstar preferem folhas jovens de carvalho e nogueira, e Peres Filho (1989) observou que folhas jovens de E. grandis são de grande importância para lagartas de T. arnobia, nos quatro primeiros ínstares. No tocante às lagartas de T. arnobia no 5 o ínstar (Tabela 3),

TABELA 1. Consumo, em 24 horas, de folhas jovens e velhas de Eucalyptus spp. por lagartas de Thyrinteina arnobia no 30 ínstar, alimentadas com Eucalyptus grandis e Eucalyptus saligna na geração anterior a $25 \pm 2^{0} \mathrm{C}, 70-80 \%$ UR e fotoperíodo de 12 horas ${ }^{1}$.

\begin{tabular}{lcccccc}
\hline \multirow{2}{*}{ Espécie } & \multicolumn{2}{c}{ Lagarta alimentada com E. grandis } & & \multicolumn{2}{c}{ Lagarta alimentada com E. saligna } \\
\cline { 2 - 3 } \cline { 5 - 6 } & Folha jovem $\left(\mathrm{cm}^{2}\right)$ & Folha velha $\left(\mathrm{cm}^{2}\right)$ & & Folha jovem $\left(\mathrm{cm}^{2}\right)$ & Folha velha $\left(\mathrm{cm}^{2}\right)$ \\
\hline E. grandis & $17,041 \mathrm{aB}$ & $0,860 \mathrm{aA}$ & & $1,688 \mathrm{aA}$ & $1,072 \mathrm{aA}$ \\
E. camaldulensis & $8,223 \mathrm{abB}$ & $0,073 \mathrm{abA}$ & & $0,827 \mathrm{abB}$ & $0,238 \mathrm{aA}$ \\
E. saligna & $4,191 \mathrm{bB}$ & $0,337 \mathrm{bA}$ & & $0,212 \mathrm{bA}$ & $0,536 \mathrm{aB}$ \\
E. citriodora & $0,384 \mathrm{bB}$ & $0 \mathrm{bA}$ & & $0,589 \mathrm{abB}$ & $0,079 \mathrm{aA}$ \\
E. robusta & $7,329 \mathrm{abB}$ & $0,437 \mathrm{abA}$ & & $0,774 \mathrm{abB}$ & $0,198 \mathrm{aA}$ \\
E. cloeziana & $8,753 \mathrm{abB}$ & $0,582 \mathrm{abA}$ & & $1,860 \mathrm{aB}$ & $0,423 \mathrm{aA}$ \\
\hline$\chi^{2}$ & 25,048 & 14,833 & & 16,786 & 9,262 \\
\hline
\end{tabular}

${ }^{1}$ Medianas seguidas da mesma letra minúscula (na coluna) e maiúscula (na linha) não diferem entre si estatisticamente $(\mathrm{P}>0,05)$.

TABELA 2. Consumo, em 24 horas, de folhas jovens e velhas de Eucalyptus spp. por lagartas de Thyrinteina arnobia no 4o ínstar, alimentadas com Eucalyptus grandis e Eucalyptus saligna na geração anterior a $25 \pm 2^{0} \mathrm{C}, 70-80 \%$ UR e fotoperíodo de 12 horas ${ }^{1}$.

\begin{tabular}{lccccc}
\hline \multirow{2}{*}{ Espécie } & \multicolumn{2}{c}{ Lagarta alimentada com E. grandis } & & \multicolumn{2}{c}{ Lagarta alimentada com E. saligna } \\
\cline { 2 - 3 } & Folha jovem $\left(\mathrm{cm}^{2}\right)$ & Folha velha $\left(\mathrm{cm}^{2}\right)$ & & Folha jovem $\left(\mathrm{cm}^{2}\right)$ & Folha velha $\left(\mathrm{cm}^{2}\right)$ \\
\hline E. grandis & $20,048 \mathrm{aB}$ & $7,256 \mathrm{aA}$ & & $6,351 \mathrm{aA}$ & $10,209 \mathrm{aB}$ \\
E. camaldulensis & $12,010 \mathrm{bB}$ & $1,218 \mathrm{bA}$ & & $2,078 \mathrm{abB}$ & $0,379 \mathrm{abA}$ \\
E. saligna & $19,279 \mathrm{abB}$ & $8,176 \mathrm{aA}$ & & $5,087 \mathrm{abA}$ & $5,809 \mathrm{aA}$ \\
E. citriodora & $9,931 \mathrm{bB}$ & $0,291 \mathrm{bA}$ & & $0,000 \mathrm{bA}$ & $0,000 \mathrm{bA}$ \\
E. robusta & $15,585 \mathrm{abB}$ & $2,013 \mathrm{abA}$ & & $1,879 \mathrm{abA}$ & $4,038 \mathrm{abB}$ \\
E. cloeziana & $15,479 \mathrm{abB}$ & $1,834 \mathrm{bA}$ & & $9,803 \mathrm{aB}$ & $4,789 \mathrm{abA}$ \\
\hline$\chi^{2}$ & 17,143 & 21,427 & 18,595 & 17,452 \\
\hline${ }^{1}$ Medianas seguidas da mesma letra minúscula (na coluna) e maiúscula (na linha) não diferem entre si estatisticamente $(\mathrm{P}>0,05)$.
\end{tabular}

TABELA 3. Consumo, em 24 horas, de folhas jovens e velhas de Eucalyptus spp. por lagartas de Thyrinteina arnobia no 5o ínstar, alimentadas com Eucalyptus grandis e Eucalyptus saligna na geração anterior a $25 \pm 2^{\circ} \mathrm{C}, 70-80 \%$ UR e fotoperíodo de 12 horas $^{1}$.

\begin{tabular}{lccccc}
\hline \multirow{2}{*}{ Espécie } & \multicolumn{2}{c}{ Lagarta alimentada com E. grandis } & & \multicolumn{2}{c}{ Lagarta alimentada com $E$ saligna } \\
\cline { 2 - 3 } \cline { 5 - 6 } & Folha jovem $\left(\mathrm{cm}^{2}\right)$ & Folha velha $\left(\mathrm{cm}^{2}\right)$ & & Folha jovem $\left(\mathrm{cm}^{2}\right)$ & Folha velha $\left(\mathrm{cm}^{2}\right)$ \\
\cline { 1 - 1 } E. grandis & $17,307 \mathrm{aA}$ & $23,675 \mathrm{aA}$ & & $28,410 \mathrm{aA}$ & $33,963 \mathrm{aA}$ \\
E. camaldulensis & $9,679 \mathrm{abA}$ & $9,613 \mathrm{bA}$ & & $10,553 \mathrm{bB}$ & $2,945 \mathrm{bA}$ \\
E. saligna & $8,408 \mathrm{abA}$ & $19,233 \mathrm{abB}$ & & $19,855 \mathrm{abA}$ & $25,171 \mathrm{aA}$ \\
E. citriodora & $0,311 \mathrm{bA}$ & $4,780 \mathrm{bB}$ & & $5,919 \mathrm{bB}$ & $1,563 \mathrm{bA}$ \\
E. robusta & $13,751 \mathrm{aA}$ & $14,592 \mathrm{bA}$ & & $15,042 \mathrm{bA}$ & $14,257 \mathrm{abA}$ \\
E. cloeziana & $17,869 \mathrm{aA}$ & $13,691 \mathrm{bA}$ & & $20,259 \mathrm{abA}$ & $14,375 \mathrm{abA}$ \\
\hline$\chi^{2}$ & 22,286 & 21,143 & & 18,762 & 24,762 \\
\hline 1 & Medianas seguidas da mesma letra minúscula (na coluna) e maiúscula (na linha) não diferem entre si estatisticamente $(\mathrm{P}>0,05)$.
\end{tabular}


não houve diferenças entre o consumo de folhas jovens e velhas das espécies utilizadas de eucalipto, com exceção de $E$. saligna, observando-se maior consumo de folhas velhas de E. grandis e E. saligna por lagartas alimentadas em $E$. grandis na geração ante-rior, e menor consumo de folhas velhas de E. camaldulensis e E. citriodora por lagartas alimentadas anteriormente, em E. saligna.

A preferência de lagartas de T. arnobia no $3-$ e e $4^{\circ}$ ínstar por folhas jovens pode estar relacionada com as propriedades físicas das folhas, pois folhas jovens são mais tenras e mais fáceis de serem raspadas e cortadas. Peres Filho (1989) observou que a raspagem e o corte de folhas jovens por lagartas de T. arnobia nos três primeiros ínstares é conseqüência da dificuldade de cortarem folhas com maior grau de dureza, o que reforça a hipótese de que a preferência alimentar por folhas jovens está relacionada com seus aspectos morfológicos. Outro aspecto importante que evidencia este fato é que tanto as folhas jovens de E. citriodora que apresentam pilosidade e as folhas velhas que são coriáceas, foram praticamente recusadas por $T$. arnobia.

$\mathrm{O}$ alimento oferecido às lagartas na geração anterior (Tabelas 1, 2 e 3) não influenciou a escolha do alimento na geração seguinte, observando-se uma tendência de maior consumo de E. grandis e menor preferência por folhas de E. citriodora.

\section{CONCLUSÕES}

1. Eucalyptus grandis é a espécie preferida por lagartas de Thyrinteina arnobia.

2. Eucalyptus citriodora é a espécie menos consumida por lagartas de Thyrinteina arnobia.

\section{REFERÊNCIAS}

ANJOS, N.; SANTOS, G.P.; ZANUNCIO, J.C. A lagarta-parda, Thyrinteina arnobia (Stoll, 1782) (Lepidoptera, Geometridae) desfolhadora de eucaliptos. Belo Horizonte: EPAMIG, 1987. p.1-56. (Boletim Técnico, n.25).
DROOZ, A.T. The elm spanworm (Lepidoptera: Geometridae): how several natural diets affect its biology. Annals Entomological Society of America, Columbus, v.63, n.2, p.391-397, 1970.

FOX, L.R.; MACAULEY,B.J. Insect grazing on Eucalyptus in response to variation in leaf tannins and nitrogen. Oecologia, Berlin, v.29, p.145-162, 1977.

HILLS, W.E. Polyphenols in the leaves of Eucalyptus L'Herit: a chemotaxonomic survey: Introduction and study of the series globulares. Phytochemistry, Elmsford, v.5, p.1075-1090, 1966.

MEYER, G.A.; MONTGOMERY, M.E. Relationships between leaf age and the food quality of cottonwood foliage for the gypsy moth, Lymantria dispar. Oecologia, Berlin, v.72, p.527-532, 1987.

OLIVEIRA, A.C.; FONSECA, E.P.; ANJOS, N.; SANTOS, G.P.; ZANÚNCIO, J.C. Resistência interespecífica de Eucalyptus spp. (Myrtaceae) à lagarta desfolhadora Thyrinteina arnobia Stoll, 1782 (Lepidoptera: Geometridae). Revista Árvore, Viçosa, v.8, p.93-103, 1984.

OSSE, L.; BRIQUELOT, A. Ocorrência de insetos em eucaliptais da Cia. Siderúgica Belgo-Mineira e combate experimental por diversos meios. Brasil Florestal, Rio de Janeiro, v.1, n.2, p.21-24, 1970.

PENFOLD, A.R.; WILLIS, J.L. The Eucalyptus. New York: Interscience, 1961. 551p.

PERES FILHO, O. Bioecologia de Thyrinteina arnobia (Stoll, 1782) (Lepidoptera, Geometridae) mantida em duas espécies de Eucalyptus (Myrtaceae). Piracicaba: ESALQ-USP, 1989. 163p. Tese de Doutorado.

RAUPP, M.J.; DENNO, R.F. Leaf age as a predictor of herbivore distribution and abundance. In: DENNO, R.F.; McCLURE, M.S. (Eds.). Variable plants and herbivores in natural and managed systems. New York: Academic Press, 1983. ch.4, p.91-124.

ZAR, J.H. Bioestatistical analysis. Englewood-Cliffs: Prentice Hall, 1984. 718p. 